\title{
Assessment of non-alcoholic fatty liver disease by non-invasive methods: present and future perspectives
}

\author{
David Vidal-González¹, Misael Uribe², Eduardo E. Montalvo-Javé2,3,4, and Natalia Nuño-Lámbarri" * \\ ${ }^{1}$ Translational Research Unit, Medica Sur Clinic and Foundation; ${ }^{2}$ Obesity and Digestive Diseases Unit, Medica Sur Clinic and Foundation; ${ }^{3}$ General \\ Surgery Service, Hepato-Pancreato-Biliary Surgery Clinic, Hospital General de Mexico; ${ }^{4}$ Department of Surgery, School of Medicine, Universidad \\ Autónoma de México. Mexico City, Mexico
}

\begin{abstract}
The difficulties that exist to assess the non-alcoholic fatty liver disease (NAFLD) prevalence and diagnosis are due to the absence of signs and symptoms in the early stages, as well as the low sensitivity of liver enzymes to identify the disease and the need to request a liver biopsy as reference standard for diagnosis despite its limitations. Non-invasive methods depend on different approaches, such as imaging techniques, including ultrasound, transient elastography, computed tomography, and magnetic resonance imaging (MRI), which can detect simple hepatic steatosis but cannot distinguish non-alcoholic steatohepatitis. Furthermore, there are biomarkers that differentiated the spectrum of the disease; these include acute phase proteins, cytokines, apoptosis, and oxidative stress markers. As well as a variety of miRNAs, which are expressed in NAFLD and could be used as blood biomarkers of progressive liver damage.
\end{abstract}

Key words: Biomarkers. Liver damage. Non-invasive methods. Steatosis.

\section{Introduction}

Non-alcoholic fatty liver disease (NAFLD) is a clinical entity characterized by the presence of hepatic fatty infiltration, without significant alcohol consumption or other chronic liver diseases caused by steatogenic drugs consumption, hepatitis $C$ virus infections, Wilson's disease, abetalipoproteinemia, parenteral nutrition, and starvation ${ }^{1}$. This disease includes a broad spectrum of liver injury, ranging from isolated steatosis to non-alcoholic steatohepatitis (NASH), fibrosis (FIB), and eventually cirrhosis ${ }^{2}$.

At present, it is a significant cause of chronic liver disease and is estimated that in some years, it will be the main reason for liver transplantation and death related to hepatic disease, especially in those with $\mathrm{NASH}^{3}$.
Its prevalence increases with age and obesity and is strongly associated with the presence of metabolic syndrome and cardiovascular diseases. It is essential to underscore that NAFLD adverse outcomes are accelerated in certain ethnic groups. The Hispanic population has been associated with the higher prevalence and the higher progression rates to NASH and $\mathrm{FIB}^{4}$. Polymorphisms in genes such as PNPLA3 can determine the differences in the disease progression ${ }^{5}$.

NAFLD estimated that the prevalence in the general population is around $25 \%{ }^{6}$ but is higher in patients with diabetes $(22-43 \%)$ and obesity $(51 \%-81 \%)^{7}$. In Mexico, a study in which patients attended a medical check-up, reports a hepatic steatosis prevalence diagnosed by ultrasound of $49.19 \%^{8}$, while another research shows

\section{Correspondence:}

*Natalia Nuño-Lámbarri

E-mail: nnunol@medicasur.org.mx;

nlambarri@gmail.com
Date of reception: 04-12-2019

Date of acceptance: 13-03-2020

DOI: 10.24875/HGMX.20000088
Available online: $21-07-2020$ Rev Med Hosp Gen Mex. 2020;83(3):135-143 www.hospitalgeneral.mx 0185-1063/@ 2020 Sociedad Médica del Hospital General de Mexico. Published by Permanyer. This is an open access article under the CC BYNC-ND license (http://creativecommons.org/licenses/by-nc-nd/4.0/). 
that patients suffering from metabolic syndrome have a $82.9 \%$ prevalence $^{9}$.

The difficulties that exist to assess NAFLD prevalence and diagnosis are due to the absence of signs and symptoms in the early stages, liver enzymes low sensitivity to designate the disease, and the need to resort to liver biopsy as a reference standard for the diagnosis despite its limitations.

Within the histopathological evaluation, two scales are mainly used to stage the findings of FIB or steatosis: The METAVIR scale and the Scheuer classification, which grade FIB in five stages: F0 (absence of FIB), F1 (portal FIB), F2 (periportal FIB), F3 (FIB bridges between portal spaces), and F4 (cirrhosis) ${ }^{10}$, and for steatosis classification a semiquantitative qualification system of four grades (from 0 to 3 ) is used, which takes into account only macrovesicular and microvesicular steatosis and evaluates the percentage of hepatocytes infiltrated by lipid vacuoles (S0, <5\%; S1, 5\%-33\%; S2, $>33 \%-66 \%$; S3, > 66\%) ${ }^{11,12}$.

Given that interventions such as liver biopsy have limitations, such as invasiveness and possible progression in steatosis severity, this process cannot be performed repeatedly, so it should be reserved only for selected patients, according to the NASH risk or more advanced stages, also patients with persistent increases in hepatic transaminase levels despite lifestyle interventions ${ }^{13}$. Furthermore, it can be associated with complications and impaired accuracy due to sampling variability because the tissue contained in a needle biopsy sample is only a small fraction of the liver, and the findings of the sample may not show what happens in the whole organ ${ }^{14,15}$.

Non-invasive methods rely on different approaches, such as imaging techniques, including ultrasonography, transient elastography, computed tomography, and magnetic resonance, which can detect simple hepatic steatosis but cannot distinguish $\mathrm{NASH}^{16}$. In addition to different biomarkers that can diagnose and differentiate NAFLD from NASH and FIB, which include acute phase proteins, cytokines, apoptosis, and oxidative stress markers. Furthermore, a variety of miRNAs are expressed in NAFLD and could be used as blood biomarkers of progressive liver injury; for example, hepatic and serum levels of miRNA-122 have been associated with steatosis, NASH, and $\mathrm{FIB}^{17}$.

\section{Laboratory studies}

Non-invasive tests such as serum biomarkers could be used as screening tools to identify patients with liver steatosis (such as, the fatty liver index [FLI], hepatic steatosis index [HSI], and SteatoTest) for diagnosing or staging FIB (such as NAFLD FIB score [NFS], BAARD score, BAAT score, and FIB-4), and also for NASH diagnosis (for example, circulating keratin 18 fragments). This scoring system has several advantages, including its high applicability, reproducibility between laboratories, and its widespread availability for non-patented models, since these scores occupy routine laboratory tests (Table 1).

\section{Steatosis biomarkers}

Many scores have been developed for steatosis detection, such as the FLI, the HS), the NAFLD liver fat score (NAFLD-LFS), and a patented algorithm, the SteatoTest ${ }^{\circledR}$.

FLI includes four variables; body mass index (BMI), waist circumference, triglycerides, and gamma-glutamyl transferase (GGT), ranging from 0 to 100 . Using ultrasound as a reference in 216 subjects with and 280 without suspected liver disease, this group demonstrates that a $\mathrm{FLI}<30$ rules out the presence of steatosis with a sensitivity of $87 \%$ while a FLI $\geq 60$ rules in the presence of steatosis with a specificity of $86 \%$. These scores were validated with liver ultrasound and nuclear magnetic resonance with spectroscopy ${ }^{18}$.

HSI was developed in a cross-sectional study with 10,724 health check-up subjects (5362 cases with NAFLD vs. age- and sex-matched controls), also using the ultrasound as a reference, and is composed of three variables; aspartate aminotransferase (AST)/alanine aminotransferase (ALT) ratio, BMI, and the presence of diabetes. HSI reported an area under the receiver operating curve (AUROC) of 0.81 and a sensitivity of $93 \%$ for diagnosing steatosis at a cutoff $<30$ and a specificity of $92 \%$ for excluding steatosis at a cutoff $>36$, validated by ultrasound ${ }^{19}$.

The NAFLD-LFS combines five variables; metabolic syndrome, type 2 diabetes, fasting insulin level, fasting AST levels, and the AST/ALT ratio. It has been proposed as a predictor of increased liver fat content, using proton magnetic resonance spectroscopy as a reference in 470 subjects. The score had an AUROC of 0.87 in the estimation and 0.86 in the validation group. The optimal cutoff point of -0.640 predicted the presence of liver steatosis with a sensitivity of $86 \%$ and specificity of $71 \%^{20}$.

SteatoTest ${ }^{\circledR}$ (BioPredictive, Paris, France) is a patented algorithm for liver steatosis diagnosis, and the only serum biomarker that used liver biopsy as reference. It 
Table 1. Scores for the diagnosis of steatosis and liver fibrosis.

\begin{tabular}{|c|c|c|c|c|c|c|c|}
\hline SCORE & Components and Equation & Cut points & Se $\%$ & $\mathbf{S p} \%$ & PPV\% & NPV\% & AUC \\
\hline Steatosis & BMI, PA, TG, GGT & $<30$ & 87 & 64 & & & 0.84 \\
\hline FLI & $\begin{array}{l}\mathrm{FLI}=\left(e 0.953^{*} \text { loge (triglycerides) }+0.139^{*} \mathrm{IMC}+0.718^{*} \text { loge }(\mathrm{GGT})\right. \\
\left.+0.053^{*} \text { abdominal girth- } 15.745\right) /\left(1+e 0.953^{*} \text { loge (triglycerides }\right)+ \\
\left.0.139^{*} \mathrm{BMI}+0.718^{*} \operatorname{loge}(\mathrm{GGT})+0.053^{*} \text { abdominal girth }-15.745\right)^{*} 100\end{array}$ & $\geq 60$ & 61 & 86 & & & \\
\hline & AST/ALT, IMC, diabetes & $<30$ & 93 & 40 & & 84 & 0.81 \\
\hline HSI & $\mathrm{HSI}=8^{*} \mathrm{ALT} / \mathrm{AST}+\mathrm{BMI}(+2 ;$ if $\mathrm{DM} 2 ;+2$; if woman) & $>36$ & 46 & 92 & 86 & & \\
\hline $\begin{array}{l}\text { NAFLD- } \\
\text { LFS }\end{array}$ & $\begin{array}{l}\text { SM, DM2, fasting insulin level, fasting AST level and AST/ALT. } \\
\text { NAFLD-LFS: }-2.89+1.18 * \text { MS (yes }=1 / \text { no }=0)+0.45 * \text { DM2 (yes }=2 / \\
\text { no }=0)+0.15 * \text { fasting insulin }(\mathrm{mU} / \mathrm{L})+0.04 * \text { fasting AST (U/L) }- \\
0.94 * \text { AST/ALT }\end{array}$ & $>-0.640$ & 85 & 70 & & & 0.87 \\
\hline & ALT, BT, GGT, CT, TG, glucose, A2M, ApoA1, haptoglobin, age, BMI & $\geq 0.3$ & 90 & 45 & 90 & 45 & 0.80 \\
\hline SteatoTest & and gender. & $\geq 0.7$ & 39 & 81 & 71 & 81 & \\
\hline \multicolumn{8}{|l|}{ Fibrosis } \\
\hline \multirow[t]{2}{*}{ FIB-4 } & AST, ALT, platelets and age. & $<1.45$ & 67 & 71 & 38 & 90 & 0.80 \\
\hline & FIB-4= Age (years) * AST $[\mathrm{U} / \mathrm{L}] /\left(\right.$ Platelets $\left.\left[10^{9} / \mathrm{L}\right]^{*}(\mathrm{ALT}[\mathrm{U} / \mathrm{L}])^{1 / 2}\right)$ & $>3.25$ & 23 & 97 & 65 & 82 & \\
\hline \multirow[t]{2}{*}{ NFS } & AST/ALT, platelets, albumin, diabetes, age, BMI and gender. & $<-1.455$ & & & & & \\
\hline & $\begin{array}{l}\mathrm{NFS}=-1.675+0.037 * \text { Age }(\text { years })+0.094 * \mathrm{IMC}\left(\mathrm{kg} / \mathrm{m}^{2}\right)+1.13^{*} \\
\mathrm{GAA} / \text { diabetes } \\
(\text { yes }=1, \text { no }=0)+0.99 * \mathrm{AST} / \mathrm{ALT}-0.013^{*} \text { platelets }\left(10^{9} / \mathrm{L}\right)-0.66^{*} \\
\text { albumin }(\mathrm{g} / \mathrm{dl})\end{array}$ & $>0.676$ & 72 & 70 & $\begin{array}{l}56 \\
90\end{array}$ & $\begin{array}{l}93 \\
85\end{array}$ & $\begin{array}{l}0.88 \\
0.82\end{array}$ \\
\hline \multirow[t]{2}{*}{ BAAT } & BMI, aged, ALT and TG. & $0-1$ & 100 & 47 & 45 & 100 & $0.71-0.86$ \\
\hline & $\begin{array}{l}\left.\text { BMI ( } \geq 28 \mathrm{Kg} / \mathrm{m}^{2}\right) \text {, age ( } 50 \text { years), ALT ( } \geq 2 \text { normal) and serum } \\
\text { triglycerides }(\geq 150 \mathrm{mg} / \mathrm{dL} \text { ) }\end{array}$ & 4 & 14 & 100 & 100 & 73 & 0.81 \\
\hline BARD & $\begin{array}{l}\text { BMI, AST/ALT, diabetes. } \\
\text { (BMI> } 28=1 \text { point, AST/ALT }>0.8=2 \text { points, } D M=1 \text { point) }\end{array}$ & 2-4 & 76 & 61 & 27 & 96 & 0.81 \\
\hline FibroTest & TB, GGT, A2M, ApoA1, haptoglobin, BMI, gender and age. & $\begin{array}{l}0.30 \\
0.70\end{array}$ & 81 & 56 & $\begin{array}{l}71 \\
97\end{array}$ & $\begin{array}{l}98 \\
89\end{array}$ & 0.88 \\
\hline Fibrometer & AST, ALT, platelets, ferritin, glucose, age, weight. & 0.71 & 79 & 61 & & & 0.94 \\
\hline
\end{tabular}

ALT: alanine aminotransferase; AST: aspartate aminotransferase; ApoA1: apolipoprotein A-1; AUC: area under the curve; A2M: a2-macroglobulin; BMI: body mass index, FLI: fatty liver index; GGT: gamma-glutamyl transferase; HIS: hepatic steatosis index; MS: metabolic syndrome; NAFLD-LFS: NAFLD Liver Fat Score; NFS: NAFLD fibrosis score; NPV: negative predictive value; PPV: positive predictive value; Se: sensitivity; Sp: specificity; TB: total bilirubin; TC: total cholesterol; TG: triglycerides; WC: waist circumference.

has been developed in a cohort with various causes of chronic liver disease, chronic hepatitis $C$, chronic hepatitis $B$, and alcoholic liver disease. This algorithm range from 0 to 1 , combines 12 parameters including age, gender, BMI, ALT, $\alpha 2$-macroglobulin, apolipoprotein A-1, haptoglobin, total bilirubin, GGT, total cholesterol, triglycerides, and glucose. In the initial study, the AUROC value for steatosis diagnosis was 0.80 , with $90 \%$ sensitivity at a 0.3 cutoff and $88 \%$ specificity at a 0.7 cutoff $^{21}$. This test has been validated with liver biopsy in 494 patients with severe obesity ${ }^{22}$.

\section{NASH biomarkers}

For NASH diagnosis, many serum biomarkers have been investigated, but cytokeratin 18 (CK-18), a physiological degradation marker is now validated. CK-18 fragments come from hepatocytes apoptosis mediated by the enzyme caspase 3 and can be measured in serum by immunoassay. Circulating serum levels of CK-18 to predict NASH in patients with NAFLD reported
AUROC of 0.83 , the sensitivity of 0.75 , and specificity of 0.81 for a CK-18 value of about $250 \mathrm{U} / \mathrm{L}$, in the initial study of validation ${ }^{23}$.

\section{FIB biomarkers}

In the evaluation of liver FIB, scoring systems have generally demonstrated more utility in advanced FIB detection than early stages. Patients with FIB require management to avoid or treat the complications of the disease (Fig. 1). The liver FIB scores that have been proposed for the detection and staging includes the FIB-4 test, NFS, BARD score, the BAAT score, and two patented algorithms, the FibroTest, and FibroMeter.

The FIB-4 test developed in patients with HIV/HCV coinfection combines platelet count, age, and ALT to asses FIB. At a cutoff of $<1.45$ in the validation set, the negative predictive value to exclude advanced FIB was $90 \%$, with a sensitivity of $70 \%$. A cutoff of $>3.25$ had a positive predictive value of $65 \%$ and a specificity of $97 \%$. 


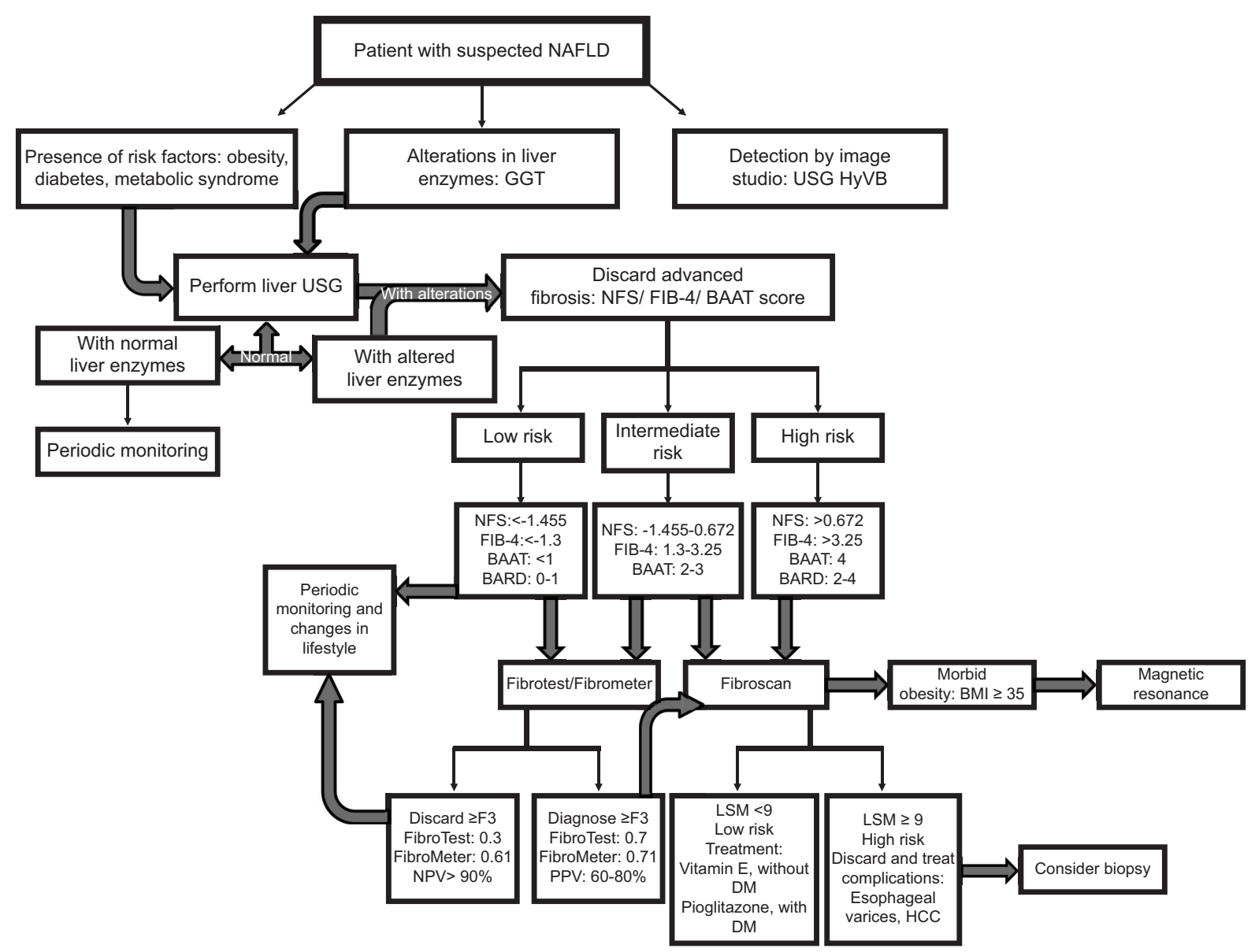

Figure 1. Algorithm suggested for the use of non-invasive tests in risk stratification of patients with suspected non-alcoholic fatty liver disease.

The NFS has been developed in a group of 733 patients with NAFLD, confirmed by liver biopsy, using six variables; age, hyperglycemia, BMI, platelet count, albumin, and AST/ALT ratio; which were significantly associated with severe FIB or cirrhosis in patients with HGNA confirmed by biopsy. This scoring system had an AUROC of 0.88 and 0.82 in the estimation and validation groups, respectively. Using the low cutoff score of -1.455 , advanced FIB could be excluded with a negative predictive value of $93 \%$ and $88 \%$ in the estimation and validation groups, respectively. Applying the high cutoff score of 0.676 , advanced FIB presence could be diagnosed with a positive predictive value of $90 \%$ and $82 \%$ in the estimation and validation groups, respectively ${ }^{25}$.

The BARD score, performed on patients diagnosed with proven liver biopsy of NAFLD, is an algorithm that combines three variables: BMI, AST/ALT ratio, and the presence of diabetes into a weighted sum $(\mathrm{BMI}>28=1$ point,
AST/ALT ratio of $>0.8=2$ points, diabetes $=1$ point), to finally generate a score from 0 to 4 . A score of 2-4 was associated with an OR for advanced FIB of 17 and a negative predictive value of $96 \%{ }^{26}$.

The BAAT score was developed in a group of 93 patients with a BMI higher than $25 \mathrm{~kg} / \mathrm{m}^{2}$, using four variables, the presence of each variable gives 1 point in the combined score: age $\geq 50$ years, $\mathrm{BMl} \geq 28 \mathrm{~kg} / \mathrm{m}^{2}$, and triglycerides $\geq 1.7 \mathrm{mmol} / \mathrm{L}$. A total score of 0 or 1 had a $100 \%$ negative predictive value for septal FIB, the sensitivity of $100 \%$ for a specificity of $47 \%$. In contrast, a score of 4 , present in only four patients, all of whom with septal FIB, gave a sensitivity of $14 \%$ and a specificity of $100 \%$ and a positive predictive value of $100 \%$ for septal FIB detection ${ }^{27}$.

FibroTest ${ }^{\circledR}$ (BioPredictive, Paris, France) is a patented panel for the detection of FIB in NAFLD, combining five biochemical markers; haptoglobin, a2-macroglobulin, apolipoprotein A1, total bilirubin, and GGT, 
corrected for age and gender, a mean standardized AUROC of 0.84 for advanced FIB in patients with NAFLD was demonstrated ${ }^{28}$.

FibroMeter $^{\circledR}$ (Echosens, Paris, France) is another commercial algorithm, which was initially developed for staging FIB in patients with viral hepatitis, the AUROC for stages F2-F4 was 0.883 , compared with 0.808 for the FibroTest, and combines seven variables; age, weight, fasting glucose, AST, ALT, ferritin, and platelet count ${ }^{29}$

A recent meta-analysis based on 64 studies in 13,046 NAFLD patients, comparing APRI, FIB-4, BARD score, NFS, FibroScan M probe, XL probe, SWE, and MRE for diagnosing advanced FIB reported summary AUROC's values of $0.77,0.84,0.76,0.84,0.88,0.85,0.95$, and 0.96 , respectively ${ }^{30}$.

\section{Imaging techniques}

Imaging is an essential tool when it comes to NAFLD diagnosis and is mainly requested when there is clinical doubt since the patient has obesity, type 2 diabetes mellitus or hyperlipidemia, also when the patient has abnormal liver function tests or when there are irregular findings in imaging studies done for other reasons (Table 2).

\section{Ultrasonography}

Abdominal ultrasound is the most used imaging method for steatosis detection since it is widely available, well-tolerated, and cheap, but its diagnostic sensitivity is lower when there are mild degrees of liver fat infiltration. Typical ultrasonography features of fatty infiltration are based on visual assessment of the echogenicity intensity; where Grade 0 presents a normal echogenicity; Grade 1 a slight and diffuse increase in echogenicity in the hepatic parenchyma, with diaphragm and intrahepatic vessel borders standard visualization; Grade 2 a moderate and diffuse increase in fine echoes, with intrahepatic vessels impaired visualization; and Grade 3 a marked increase in echogenicity, with poor or no visualization of the intrahepatic vessel borders and diaphragm ${ }^{31}$.

The ultrasound has a high accuracy in fatty liver diagnosis when the total area of hepatic steatosis exceeds $20 \%$, with a sensitivity of $96 \%$, but decreases when there is a fat infiltration of $5 \%$ at a sensitivity of $82 \%{ }^{32}$, in addition to the intraobserver and interobserver variability reported in several studies as a dependent operator too ${ }^{33}$. Severe obesity may also limit the ability to reliably detect liver echogenicity due to thick layers of subcutaneous fat. In a study that evaluated NAFLD in obese patients with an average BMI of 34.1 $\mathrm{kg} / \mathrm{m}^{2}$, it shows a low sensitivity of $63 \%$ was found, taking into account only the echogenicity of the hepatic parenchyma ${ }^{34}$. On the other hand, a meta-analysis that included 34 studies, with 2815 patients to which underwent an ultrasound, it is observed that around 20 $30 \%$ have hepatic steatosis with a sensitivity of $84.38 \%$ and a specificity of $93.6 \%$, taking liver biopsy as a reference ${ }^{35}$.

There are limited data available that support the use of ultrasound as a screening tool; however, the clinical criterion is essential to use it as a first-line diagnostic test, mainly in patients with risk factors and abnormal liver enzymes, especially GGT ${ }^{36}$. Nevertheless, due to its low cost, safety, and accessibility, ultrasound is probably the imaging technique of choice for fatty liver detection in clinical centers, as recommended by the European guidelines for the management of NAFLD in patients at risk ${ }^{37}$.

\section{Transient elastography}

Transient elastography was developed to quantify the liver rigidity by vibrations of low amplitude and low frequency that are transmitted by the transducer, which induce an elastic cutting wave that propagates through the hepatic tissue. Pulse-echo ultrasound acquisition is used to follow the propagation of the wave and to measure its velocity, which is directly related to tissue stiffness. The more rigid the tissue, the faster the shear wave propagates, allowing the degree of liver FIB to be determined, with results that are expressed in kilopascals $(\mathrm{kPa})$, and range from 2.5 to $75 \mathrm{kPa}$ with average values around $5 \mathrm{kPa}$, higher in patients with elevated $\mathrm{BMI}$ or metabolic syndrome ${ }^{38}$. At the same time, it evaluates the degree of steatosis determined by the controlled attenuation parameter (CAP) through an algorithm that calculates the ultrasound signal attenuation and is expressed in $\mathrm{dB} / \mathrm{m}$, with ranges of $100-400$ $\mathrm{dB} / \mathrm{m}$, and a median for each degree of steatosis of: 205 (180-227) dB/m for S0; 245 (231-268) dB/m for S1; 299 (268-323) dB/m for S2; and 321 (301-346) dB/m for S3, according to one of the first cohorts for its validation, in patients with chronic liver disease due to various causes ${ }^{39}$. However, these values can be influenced by other factors such as BMI increase or other comorbidities such as diabetes, as reported by Karlas et al. in a meta-analysis that included 2735 patients, where it was observed that CAP values were influenced 


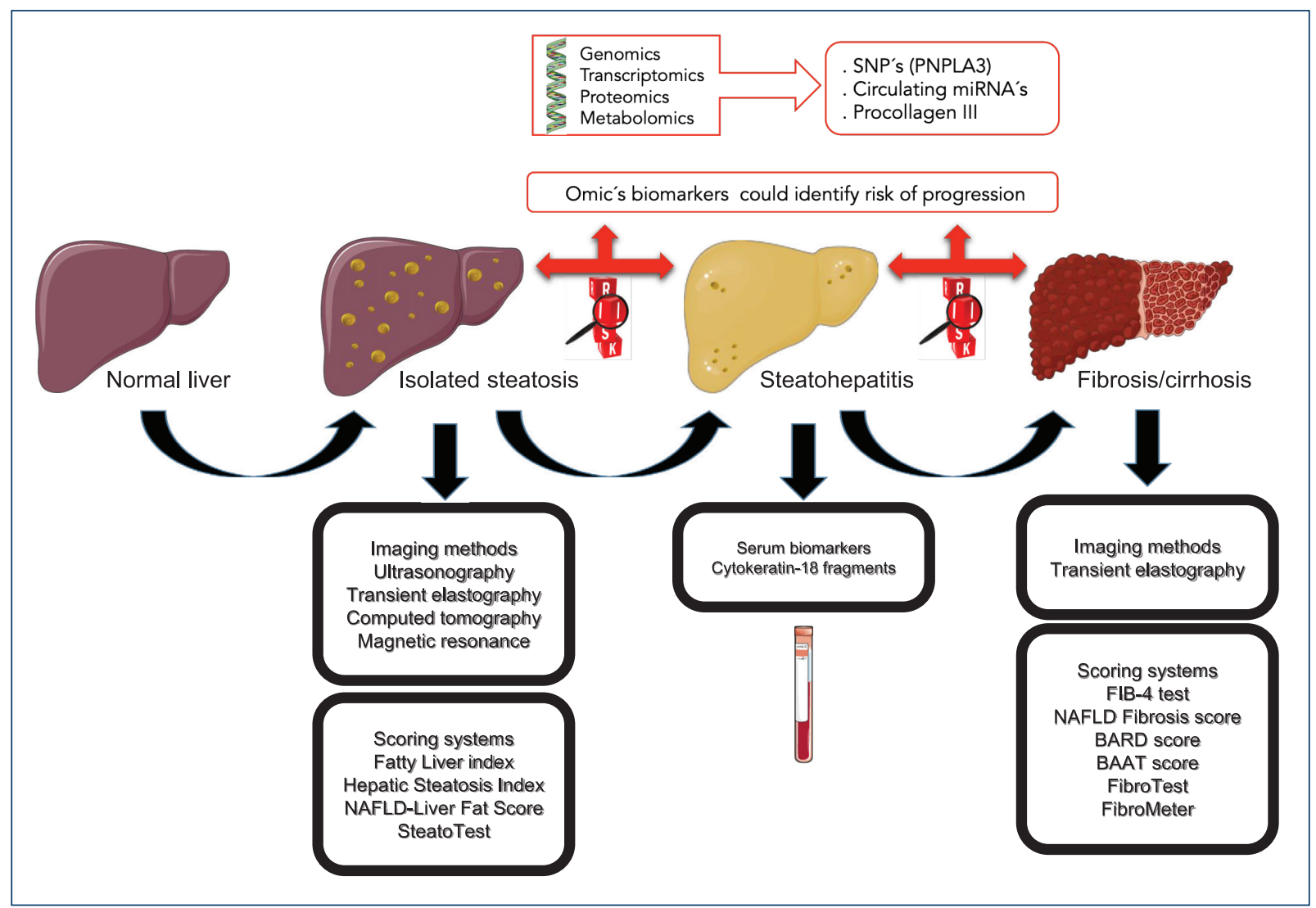

Figure 2. Diagnostic techniques to assess non-alcoholic fatty liver disease progression.

Table 2. Advantages and disadvantages of imaging techniques for fatty liver disease evaluation

\begin{tabular}{|l|l|l|l|}
\hline Diagnostic tool & Advantage & Disadvantages & Clinical application \\
\hline Ultrasound & High availability and low cost & $\begin{array}{l}\text { Operator dependent, does not distinguish between } \\
\text { fibrosis and steatosis, not useful in mild degrees, in } \\
\text { addition to having low specificity }\end{array}$ & First diagnostic tool \\
\hline $\begin{array}{l}\text { Transient } \\
\text { elastography }\end{array}$ & $\begin{array}{l}\text { Evaluate both steatosis and } \\
\text { fibrosis at the same time, and } \\
\text { is simple and fast }<5 \mathrm{~min})\end{array}$ & $\begin{array}{l}\text { Not very accessible, the cut points are not very well } \\
\text { defined }\end{array}$ & $\begin{array}{l}\text { Useful to discard advanced } \\
\text { fibrosis or early stages of } \\
\text { steatosis }\end{array}$ \\
\hline $\begin{array}{l}\text { Computed } \\
\text { tomography }\end{array}$ & It is not operator-dependent & Radiation exposure & $\begin{array}{l}\text { Diagnosis of steatosis in the } \\
\text { liver study for other causes }\end{array}$ \\
\hline $\begin{array}{l}\text { Magnetic } \\
\text { resonance }\end{array}$ & High specificity & Low availability and high cost & $\begin{array}{l}\text { Useful in clinical trials and in } \\
\text { patients with morbid obesity }\end{array}$
\end{tabular}

by an estimated change of $10 \mathrm{~dB} / \mathrm{m}$ (3.5-16) for diabetics and $4.4 \mathrm{~dB} / \mathrm{m}$ (3.8-5.0) per unit of $\mathrm{BMI}$, in this study, the optimal cutoff points were $248 \mathrm{~dB} / \mathrm{m}(237-261)$ and $268 \mathrm{~dB} / \mathrm{m}$ (257-284) for those above S0 and S1, respectively ${ }^{40}$.

Transient elastography is performed with the patient in a supine position, with the right arm in maximal abduction, placed behind the head to facilitate access to the right hepatic lobe through an intercostal space, where the tip of the transducer is placed; then, the operator presses the probe button to start the measurements that quantify an approximate volume of $1 \mathrm{~cm}$ wide and $4 \mathrm{~cm}$ long, this area is at least 100 times bigger than a biopsy sample, and therefore, more 
representative of the hepatic parenchyma ${ }^{41}$. The depth of the measurement is between 25 and $65 \mathrm{~mm}$ with the $M$ probe, and between 35 and $75 \mathrm{~mm}$ with the XLprobe, the probe will be chosen as suggested by the automatic device selection tool ${ }^{42}$. At least, ten successful acquisitions should be performed on each patient, only the results obtained with a success rate of at least $60 \%$ and an interquartile range (IQR) $\leq 30 \%$ of the mean liver stiffness measurement value (IQR/liver stiffness measurement $\leq 30 \%)^{43}$, and an IQR of the CAP $\leq 40$ $\mathrm{dB} / \mathrm{m}$ should be considered reliable ${ }^{44}$.

An accuracy variation for diagnosis and staging has also been reported through the use of the "XL" probe, developed for obese patients evaluation. In a study in which 210 patients were evaluated for FIB degree by transient elastography using the $\mathrm{XL}$ probe compared to biopsy, a discrepancy was observed in the staging of FIB in $11 \%(n=24)$, not attributable to age, sex, and presence of diabetes or hepatitis $B$ or $C$ virus infection. In the presence of morbid obesity, a 4-5 times higher discordance was found regarding a BMI $\geq 40$; however, it was less likely to follow the valid measurement criteria as described previously ${ }^{45}$. Furthermore, in a recent prospective multicenter study, which evaluated the accuracy to diagnose steatosis and liver stiffness through transient elastography, using biopsy as the standard of reference, showed that in 450 patients studied for suspected NAFLD, reported AUROC values that vary from 0.70 to 0.89 . The CAP accuracy was highest at a stage of $S 1$, with an AUROC of 0.87 , a sensitivity of 0.80 , and a specificity of 0.83 at a threshold of $302 \mathrm{~dB} / \mathrm{m}$ selected by maximizing Youden's index. For the S2 stage, accuracy dropped to an area under the curve of 0.77 with the corresponding sensitivity of 0.70 and specificity of 0.76 with an AUROC of 0.70 , while for stage S3 had a sensitivity of 0.72 and a specificity of $0.63^{46}$.

On the other hand, for liver stiffness, the accuracy was highest at the F4 threshold, with an AUROC of 0.89 , a sensitivity of 0.85 , and a specificity of 0.79 at a threshold of $13.6 \mathrm{kPa}$ selected by maximizing Youden's index. The accuracy was lower at the lower FIB thresholds, dropping to an AUROC of 0.80 for F3 with a sensitivity of 0.71 and a specificity of 0.75 at a threshold of $9.7 \mathrm{kPa}$. For F2 there is an AUROC of 0.77 , with a sensitivity of 0.71 and a specificity of 0.70 at a threshold of $8.2 \mathrm{kPa}$. The type of probe or the steatosis did not affect the liver stiffness; the only parameter that significantly affected the liver stiffness was the FIB stage. The cutoff values for CAP staging S1, S2, and S3 were $302 \mathrm{~dB} / \mathrm{m}, 331 \mathrm{~dB} / \mathrm{m}$, and $337 \mathrm{~dB} / \mathrm{m}$, respectively. In the case of liver stiffness, the cutoff values for F2, F3, and F4 were $8.2 \mathrm{kPa}, 9.7 \mathrm{kPa}$, and $13.6 \mathrm{kPa}$, respectively ${ }^{46}$.

Therefore, it can be observed that the ideal applicability of transient elastography would be in a patient without morbid obesity, with risk factors to detect initial degrees of fatty infiltration or in the follow-up of patients with chronic liver disease to rule out severe FIB or cirrhosis.

\section{Acoustic radiation force impulse (ARFI)}

It is an imaging method where tissue compression is not performed manually, but by an ARFI, which deforms the tissue, after the pressure pulse is applied, the displacement is measured. It consists on pulsed beams generation by means of long-lasting trains to create radiation forces that cause displacements located within the tissue to measure a quantitative shear-wave velocity $(\mathrm{m} / \mathrm{s})$. These displacements are recorded by ultrasound, generating a map of the tissue elasticity, without using an external force ${ }^{47}$.

This process is repeated for all the scan lines so that two images, pre- and post-compression, are obtained, which are processed using a cross-correlation algorithm, with which it will be possible to compute the tissue position differences in each point along the axial axes, between the states of relaxation and compression. The amount of displaced tissue reflects its elasticity and will be directly proportional to the magnitude of the force applied and inversely proportional to the stiffness of the tissue ${ }^{48}$.

\section{Computed tomography}

Computed tomography evaluates steatosis through the reduction in liver attenuation, which can be measured in Hounsfield units (HU) and appears as hypodense liver parenchyma. The attenuation value in a healthy liver is around 50-57 $\mathrm{HU}$ without contrast, which is reduced due to the lipid overload in the liver that facilitates the appropriate determination of the steatosis degree, reaching a specificity of $100 \%$ when the fat content is higher than $30 \%{ }^{49}$. There are five criteria to diagnose steatosis for non-contrast computed tomography: hepatic attenuation $\leq 40 \mathrm{HU}$, liver attenuation less than spleen attenuation minus $10 \mathrm{HU}$, hepatic attenuation less than or equal to spleen attenuation, liver attenuation less than or equal to spleen attenuation plus $5 \mathrm{HU}$, and liver to spleen attenuation ratio $\leq 1.1$. These vary in specificity and sensitivity, reaching higher values when the fat content is higher than $30 \%$; 
however, there is a lack of histological correlation, without being able to exclude the coexistence of NASH or early cirrhosis. Furthermore, possibly an iron deposit could mask some cases of steatosis. Due to the above, in addition to the emitted radiation and high cost, it does not allow its frequent use ${ }^{50}$.

\section{MRI}

MRI is the most accurate method for the detection and quantification of hepatic fat content, the hepatic steatosis detection in MRI is based on the images of chemical change, the protons in water and fat may be in two modes, in-phase and out of phase. Fat liver content caused a decrease in hepatic signal intensity in the out of phase modality in comparison with the "in phase" images, being able to measure the degree of fat infiltration from this difference in intensity. MRI is not suitable as a screening method due to high costs and limited comparability between different MRI techniques ${ }^{51}$.

\section{Conclusions}

Today, there are several non-invasive methods to evaluate and diagnose fatty liver disease, which are very helpful for the attending physician, and they can be used according to the resources of the different care centers.

In the first instance, when there is a patient with suspected fatty liver, the first thing that is suggested is to look for associated comorbidities such as the presence of obesity, metabolic syndrome, diabetes, or abnormal liver function tests. In case of alterations, it is proposed to perform a liver ultrasound, being a cost-effective imaging technique in primary care, if it comes out with alterations it is advisable to prepare some biochemical test to rule out liver FIB. If this evaluation comes out with modifications, the next step would be to continue with an imaging technique, either transition elastography, which has the advantage of evaluating both steatosis and FIB degree at the same time or MRI that is the most accurate imaging method to quantify fatty liver disease, since it can differentiate between NASH and non-progressive NAFLD. Until now, the best marker in blood to detect NASH is CK18 , however to identify liver FIB there are several tests and algorithms that are quite useful. Finally, in case the physician still has uncertainty, he could request a liver biopsy (Figs. 1 and 2).

The suggested that algorithm for the use of non-invasive tests in patients with suspected NAFLD is mainly for first-contact physicians, since an early diagnosis of liver steatosis could slow the progression and therefore the prevalence of the disease, as well as minimize costs. The algorithm also offers multiple options for both biochemical and imaging tools that are affordable for all types of patients or health centers.

On the other hand, liver diseases genomics and transcriptomics have changed dramatically in the past decade, aided by the emergence of technologies such as microarrays and massive sequencing; however, studies on epigenetic factors expression and their functional effect on NAFLD development are still missing; therefore, it would be of great interest to identify and characterize the differential expression profile of epigenetic factors to look for a molecular signature or a biomarker that can predict the development and severity of the disease and help to make an early diagnosis. In addition, it is desired to know the physiological microenvironment that could favor the complications in the patient's health (Fig. 2).

\section{Acknowledgments}

We appreciate the support of the General Hospital of Mexico and Medica Sur Clinic and Foundation so that this article could be made. All authors have contributed to the realization and improvement of the article, also agreed on the content of the manuscript. David Vidal-González and Natalia Nuño-Lámbarri design and wrote the article. Misael Uribe and Eduardo E. Montalvo Javé revised, contributed with diverse ideas and corrected the final version of the manuscript.

\section{Conflicts of interest}

The authors declare that not had any competing financial interests or commercial relationships that might pose a conflict of interest.

\section{Ethical disclosures}

Protection of human and animal subjects. The authors declare that no experiments were performed on humans or animals for this study.

Confidentiality of data. The authors declare that no patient data appear in this article.

Right to privacy and informed consent. The authors declare that no patient data appear in this article. 


\section{References}

1. Chalasani N, Younossi Z, Lavine JE, Charlton M, Cusi K, Rinella M, et al The diagnosis and management of nonalcoholic fatty liver disease: practice guidance from the American association for the study of liver diseases. Hepatology. 2018;67:328-57.

2. Farrell GC, Larter CZ. Nonalcoholic fatty liver disease: from steatosis to cirrhosis. Hepatology. 2006;43:S99-112.

3. Charlton MR, Burns JM, Pedersen RA, Watt KD, Heimbach JK, Dierkhising RA. Frequency and outcomes of liver transplantation for nonalcoholic steatohepatitis in the United States. Gastroenterology. 2011;141:1249-53.

4. Satapathy SK, Sanyal AJ. Epidemiology and natural history of nonalcoholic fatty liver disease. Semin Liver Dis. 2015;35:221-35.

5. Daly AK, Ballestri S, Carulli L, Loria P, Day CP. Genetic determinants of susceptibility and severity in nonalcoholic fatty liver disease. Expert Rev Gastroenterol Hepatol. 2011;5:253-63.

6. Mundi MS, Velapati S, Patel J, Kellogg TA, Abu Dayyeh BK, Hurt RT. Evolution of NAFLD and Its Management. Nutr Clin Pract. 2020;35:72-84

7. Younossi ZM, Koenig AB, Abdelatif D, Fazel Y, Henry L, Wymer M. Global epidemiology of nonalcoholic fatty liver disease meta-analytic assessment of prevalence, incidence, and outcomes. Hepatology. 2016;64:73-84

8. Briseño-Bass P, Chávez-Pérez R, López-Zendejas M. Prevalence of hepatic steatosis and its relation to liver function tests and lipid profile in patients at medical check-up. Rev Gastroenterol Mex. 2019;84:290-5.

9. Méndez JA, Fillot AC, Gutiérrez JC, Aguado LQ, Barallat JM. Prevalence of non-alcoholic fatty liver disease and associated risk factors among managers from the community of Madrid. Arch Prev Riesgos Labor. 2014;17:84-90.

10. Bedossa $\mathrm{P}$. An algorithm for the grading of activity in chronic hepatitis $\mathrm{C}$. Hepatology. 2004;24:289-93.

11. Kleiner DE, Brunt EM, Van Natta M, Behling C, Contos MJ, Cummings OW, et al. Design and validation of a histological scoring system for nonalcoholic fatty liver disease. Hepatology. 2005;41:1313-21.

12. Scheuer PJ. The nomenclature of chronic hepatitis: time for a change. $J$ Hepatol. 1995;22:112-4

13. Nalbantoglu I, Brunt EM. Role of liver biopsy in nonalcoholic fatty liver disease. World J Gastroenterol. 2014;20:9026-37.

14. Ratziu V, Charlotte F, Heurtier A, Gombert S, Giral P, Bruckert E, et al Sampling variability of liver biopsy in nonalcoholic fatty liver disease. Gastroenterology. 2005;128:1898-906.

15. Bedossa $P$, Dargère $D$, Paradis V. Sampling variability of liver fibrosis in chronic hepatitis C. Hepatology. 2003;38:1449-57.

16. Saadeh S, Younossi ZM, Remer EM, Gramlich T, Ong JP, Hurley M et al. The utility of radiological imaging in nonalcoholic fatty liver disease. Gastroenterology. 2002;123:745-50.

17. Pirola CJ, Gianotti TF, Castaño GO, Mallardi P, Martino JS, Ledesma MM et al. Circulating microRNA signature in non-alcoholic fatty liver disease: from serum non-coding RNAs to liver histology and disease pathogenesis. Gut. 2015;64:800-12.

18. Bedogni G, Bellentani S, Miglioli L, Masutti F, Passala M, Castiglione A et al. The fatty liver index: a simple and accurate predictor of hepatic steatosis in the general population. BMC Gastroenterol. 2006;6:33.

19. Lee JH, Kim D, Kim HJ, Lee CH, Yang JI, Kim W, et al. Hepatic steatosis index: a simple screening tool reflecting nonalcoholic fatty liver disease. Dig Liver Dis. 2010;42:503-8.

20. Kotronen A, Peltonen M, Hakkarainen A, Sevastianova K, Bergholm R Johansson LM, et al. Prediction of non-alcoholic fatty liver disease and liver fat using metabolic and genetic factors. Gastroenterology. 2009;137:865-72

21. Poynard T, Ratziu V, Naveau S, Thabut D, Charlotte F, Messous D, et al. The diagnostic value of biomarkers (Steato Test) for the prediction of liver steatosis. Comp Hepatol. 2005;4:10.

22. Poynard T, Lassailly G, Diaz E, Clement K, Caïazzo R, Tordjman J, et al. Performance of biomarkers fibrotest, actitest, steatotest, and nashtest in patients with severe obesity: meta analysis of individual patient data. PLoS One. 2012;7:e30325.

23. Feldstein AE, Wieckowska A, Lopez AR, Liu YC, Zein NN, McCullough AJ. Cytokeratin-18 fragment levels as noninvasive biomarkers for nonalcoholic steatohepatitis: a multicenter validation study. $\mathrm{He}-$ patology. 2009;50:1072-8

24. Sterling RK, Lissen E, Clumeck N, Sola R, Correa C, Montaner J, et al. Development of a simple noninvasive index to predict significant fibrosis in patients with HIV/HCV coinfection. Hepatology. 2006;43:1317-25.

25. Angulo P, Hui JM, Marchesini G, Bugianesi E, George J, Farrell GC et al. The NAFLD fibrosis score: a noninvasive system that identifies liver fibrosis in patients with NAFLD. Hepatology. 2007;45:846-54.

26. Harrison SA, Oliver D, Arnold HL, Gogia S, Neuschwander-Tetri BA Development and validation of a simple NAFLD clinical scoring system for identifying patients without advanced disease. Gut. 2008;57:1441-7.

27. Ratziu V, Giral P, Charlotte F, Brucket E, Thibault V, Theodorou I, et al. Liver fibrosis in overweight patients. Gastroenterology. 2000;118:1117-23.
28. Poynard $T$, Imbert-Bismut $F$, Munteanu M, Messous D, Myers RP, Thabut $D$, et al. Overview of the diagnostic value of biochemical markers of liver fibrosis (FibroTest, HCV FibroSure) and necrosis (ActiTest) in patients with chronic hepatitis C. Comp Hepatol. 2004;3:8.

29. Calès P, Oberti F, Michalak S, Hubert-Fouchard I, Rousselet MC Konaté $A$, et al. A novel panel of blood markers to assess the degree of liver fibrosis. Hepatology. 2005;42:1373-81.

30. Xiao G, Zhu S, Xiao X, Yan L, Yang J, Wu G. Comparison of laboratory test, ultrasound, or magnetic resonance elastography to detect fibrosis in patients with nonalcoholic fatty liver disease: a meta-analysis. Hepatology. 2017:66:1486-501.

31. Hamaguchi M, Kojima T, Itoh Y, Harano Y, Fujii K, Nakajima T, et al. The severity of ultrasonographic findings in nonalcoholic fatty liver disease reflects the metabolic syndrome and visceral fat accumulation. Am J Gastroenterol. 2007:102:2708-15.

32. Dasarathy S, Dasarathy J, Khiyami A, Joseph R, Lopez R, McCullough AJ. Validity of real time ultrasound in the diagnosis of hepatic steatosis: a prospective study. J Hepatol. 2009;51:1061-7.

33. Strauss S, Gavish E, Gottlieb P, Katsnelson L. Interobserver and intraobserver variability in the sonographic assessment of fatty liver. Am J Roentgenol. 2007;189:1449.

34. Bril F, Ortiz-Lopez C, Lomonaco R, Orsak B, Freckleton M, Chintapalli K, et al. Clinical value of liver ultrasound for the diagnosis of nonalcoholic fatty liver disease in overweight and obese patients. Liver Int. 2015;35:2139-46.

35. Hernaez R, Lazo M, Bonekamp S, Kamel I, Brancati F, Guallar JC Diagnostic accuracy and reliability of ultrasonography for the detection of fatty liver: a meta-analysis. 2014;54:1082-90

36. Haring R, Wallaschofski H, Nauck M, Dörr M, Baumeister SE, Völzke H. Ultrasonographic hepatic steatosis increases prediction of mortality risk from elevated serum gamma-glutamyl transpeptidase levels. Hepatology. 2009:50:1403-11.

37. Marchesini G, Roden M, Vettor R. Response to: comment to EASLEASD-EASO clinical practice guidelines for the management of non-alcoholic fatty liver disease. J Hepatol. 2017;66:466-7.

38. Castera L, Forns $X$, Alberti A. Non-invasive evaluation of liver fibrosis using transient elastography. J Hepatol. 2008;48:835-47.

39. Sasso $M$, Beaugrand $M$, de Ledinghen V, Douvin $C$, Marcellin, $P$, Poupon $\mathrm{R}$, et al. Controlled attenuation parameter (CAP): a novel VCTETM guided ultrasonic attenuation measurement for the evaluation of hepatic steatosis: preliminary study and validation in a cohort of patients with chronic liver disease from various causes. Ultrasound Med Biol. 2010;36:1825-35

40. Karlas T, Petroff D, Sasso M, Mi YQ, Lédinghen V, Kumar M, et al. Individual patient data meta-analysis of controlled attenuation parameter (CAP) technology for assessing steatosis. J Hepatol. 2017:66:1022-30.

41. Barr RG, Nakashima K, Amy D, Cosgrove D, Farrokh A, Schafer F, et al. WFUMB guidelines and recommendations for clinical use of ultrasound elastography: part 2: breast. Ultrasound Med Biol. 2015;41:1148-60.

42. de Ledinghen V, Vergniol J, Foucher J, El-Hajbi F, Merrouche W, Rigalleau V. Feasibility of liver transient elastography with FibroScan® using a new probe for obese patients. Liver Int. 2010;30:1043-8.

43. Castera L, Foucher J, Bernard PH, Carvalho F, Allaix D, Merrouche W, et al. Pitfalls of liver stiffness measurement: a 5-year prospective study of 13,369 examinations. Hepatology. 2010;51:828-35.

44. Wong VW, Petta S, Hiriart JB, Cammà C, Wong GL, Marra F, et al. Validity criteria for the diagnosis of fatty liver by $M$ probe-based controlled attenuation parameter. J Hepatol. 2017;67:577-84.

45. Myers RP, Pomier-Layrargues G, Kirsch R, Pollet A, Beatin M, Levstik M, et al. Discordance in fibrosis staging between liver biopsy and transient elastography using the FibroScan XL probe. J Hepatol. 2012;56:564-70.

46. Eddowes PJ, Sasso M, Allison M, Tsocharzis E, Anstee QM, Sheridan D, et al. Accuracy of fibroscan controlled attenuation parameter and liver stiffness measurement in assessing steatosis and fibrosis in patients with nonalcoholic fatty liver disease. Gastroenterology. 2019;156:1717-30.

47. Su S, Wang W, Nadebaum D, Nicol A, Gorelik A, Lai J, et al. Skin-liver distance and interquartile range-median ratio as determinants of interoperator concordance in acoustic radiation force impulse imaging. J Med Ultrasound. 2019;27:177.

48. Palmeri ML, McAleavey SA, Trahey GE, Nightingale KR. Ultrasonic tracking of acoustic radiation force-induced displacements in homogeneous media. IEEE Trans Ultrason Ferroelectr Freq Control. 2006;53:1300-13.

49. Bydder GM, Chapman RW, Harry D, Bassan L, Sherlock S, Kreel L. Computed tomography attenuation values in fatty liver. J Comput Tomogr. 1981;5:33-5.

50. Boyce CJ, Pickhardt PJ, Kim DH, Taylor AJ, Bruce RJ, Lindstrom MJ, et al. Hepatic steatosis (fatty liver disease) in asymptomatic adults identified by unenhanced low-dose CT. Am J Roentgenol. 2010;194:623-8.

51. Fishbein M, Castro F, Cheruku S, Jain S, Webb B, Gleason T, et al. Hepatic MRI for fat quantitation: its relationship to fat morphology, diagnosis, and ultrasound. J Clin Gastroenterol. 2005;39:619-25. 